\title{
Research Progress of Transportation Under Sharing Mode
}

\author{
Yiheng Song ${ }^{1,}$, Xinyu Dong ${ }^{2}$, Xiaowei Qin ${ }^{3}$, Jiashun Chen ${ }^{4}$ \\ ${ }^{1}$ School of Civil Engineering, Southeast University, Nanjing, China \\ ${ }^{2}$ School of Transportation, Southeast University, Nanjing, China \\ ${ }^{3}$ School of Economics \& Management, Southeast University, Nanjing, China \\ ${ }^{4}$ School of Computer Science and Engineering, Southeast University, Nanjing, China
}

\section{Email address:}

songyih@seu.edu.cn (Yiheng Song),1034293511@qq.com (Xinyu Dong),1103180278@qq.com (Xiaowei Qin), 2453821308@qq.com (Jiashun Chen)

${ }^{*}$ Corresponding author

\section{To cite this article:}

Yiheng Song, Xinyu Dong, Xiaowei Qin, Jiashun Chen. Research Progress of Transportation Under Sharing Mode. Science Discovery. Vol. 7, No. 5, 2019, pp. 257-265. doi: 10.11648/j.sd.20190705.11

Received: August 4, 2019; Accepted: September 24, 2019; Published: September 27, 2019

\begin{abstract}
In order to more effectively and accurately study the establishment of Shared transportation system, this paper summarizes the research progress of domestic and foreign scholars' Shared transportation system.On this basis, the development direction of its description and technology is prospected.The paper points out that: 1) further development of data acquisition and analysis platform of intelligent lock is the development direction of perfect standardized management; 2) proton exchange membrane fuel cell, solar energy, reluctance motor power generation and multi-energy model can be used as the new energy power of the Shared car; 3 ) to solve the problems of charging and vehicle configuration encountered in the use of Shared vehicles, a new charging pile and intelligent sharing system are proposed; 4) for various complex transportation modes and technologies, how to properly classify them, establish performance indicators, and formulate various test standards and engineering application specifications will be one of the important directions for future research.
\end{abstract}

Keywords: Sharing Mode, Transportation, Sharing Transportation

\section{共享模式下交通运输的研究进展}

\author{
宋毅恒 ${ }^{*}$, 董心宇 ${ }^{2}$, 秦小桅 ${ }^{3}$, 陈嘉顺 ${ }^{4}$ \\ 1东南大学土木工程, , 南京, 中国 \\ 2东南大学交通运输, 南京, 中国 \\ 3东南大学经济管理, 南京, 中国 \\ 4东南大学计算机科学与工程, 南京, 中国
}

\section{邮箱}

songyih@seu.edu.cn（宋毅恒）, 1034293511@qq.com（董心宇）, 1103180278@qq.com（秦小桅）,

2453821308@qq.com(陈嘉顺)

摘要: 本文为了更加有效精准地研究共享交通运输体系的建立, 综述了国内外学者共享模式下的交通运输研究进展情 况。并在此基础上, 重点对其描述、技术两方面发展方向进行了展望。文中指出, 1）进一步开发智能锁的数据获取与 分析平台是完善规范化管理的发展方向；2）质子交换膜燃料电池、太阳能、磁阻电机发电、多能源模型等可以作为共 享车的新型能源动力; 3) 针对共享车辆在使用时遇到的充电及车辆配置问题, 提出了采用新型充电桩及智能共享系统 等解决方案；4）针对各种复杂的交通运输方式及技术, 如何对它们进行适当分类、确立性能指标, 并制订出各类测试 标准及其工程应用规范等, 将是今后研究的重要方向之一。 
关键词：共享模式，交通运输，共享交通

\section{1. 前言}

在传统社会中，朋友或熟人之间物品的共用，算是初 态的共享, 这种共享以实物为主, 过程中没有产生报酬和 利润[1]。2000年之后, 互联网web2.0时代的到来, 随着 BBS、论坛的出现, 用户们开始在网络平台上与陌生人分 享信息。2010年前后, 国外Uber、Airbnb, 国内滴滴打车、 快的打车、小猪短租等一系列实物共享平台的出现, 再到 之后ofo、摩拜等共享单车企业的成立[2], 共享经济正式 进入大众视野, 它已经渗透到了交通、住宿、金融、餐饮 等众多领域。

在共享经济全球化的背景下, 共享自行车是“最后1公 里”出行的解决方案 [3], 但是它在 $2 \mathrm{~km}$ 以上的出行上无能 为力。因此共享电单车得以借机进行推广, 方便市民出行 的同时, 解决城市拥堵和环境污染问题。对于共享经济和 共享交通, 国内外学者都有诸多研究。2009年, YL Ke等 [4]为电动自行车研发了双象限直流电动机驱动器, 以控制 下坡行驶速度。2013年, G Zervas[5]等认为共享经济可能 会改变社会福利; L Caggiani等[6]提出了动态自行车再分 配过程的仿真模型；2015年, 汤天波等[7]认为共享经济是 “互联网+”下的颠覆性经济模式; 卢希鹏[8]从时间角度提 出了共享经济中的“随经济”战略思维; 孟凡新 [9]以淘宝平 台的运作模式为例提出了网络交易市场机制的理论分析 框架。2016年, 李强治[10]认为共享经济的核心在于解决 个体效率构架; Kai-way Chu等[11]提出了一种基于微控制 器的现代城市自行车共享系统物联网解决方案; 陈元志 [12]提出政府部门应该采用创新友好型监管制度; 2017年,
SG Mason[13]以零售引力模型及其衍生物为基础, 为美国 城市中的自行车共享位置开发了可复制的模型; 刘蕾等 [14]认为传统经济模式到共享经济模式的转变本质是一个 “去中介化”和“再中介化”的过程; J Chen等[15]设计了一种 基于蓝牙低功耗和GPRS通信的智能锁系统; 2018年，陶 雅洁[16]构建了关于共享单车市场饱和度模型及模糊综合 评价模型; 毛方强等 [17]实现了基于公共自行车系统的电 助力分离模式; 黄砷才等 [18]提出了基于RFID技术的电单 车防盗系统; 彭华涛等 [19]基于共享经济模仿行为存在内 在逻辑提出了“三制三监督”的协同治理机制。

限于篇幅, 为了能够对所涉及的内容作较为全面的阐 述, 本文重点阐述了共享体系的分类标准, 并深入探讨共 享交通工具的能源动力、组织与管理, 另外还揭示了现存 的问题, 同时指出发展前景。希望能为共享大背景下的交 通运输研究抛砖引玉。

\section{2. 共享体系的分类}

共享交通是共享经济在交通领域的表现形式，一般是 指通过市场机制, 以获得一定报酬为主要目的, 基于陌生 人且存在交通工具使用权暂时转移的一种新的交通服务 供给模式。共享交通的类型因分类标准而异, 为了便于在 有关研究或应用中可以根据各自的需要、采用相应的分类 标准，本文根据原始交通工具的分类、使用空间、是否需 要固定停车点的分类标准分类, 以下简介共享交通工具的 类型(图1)。

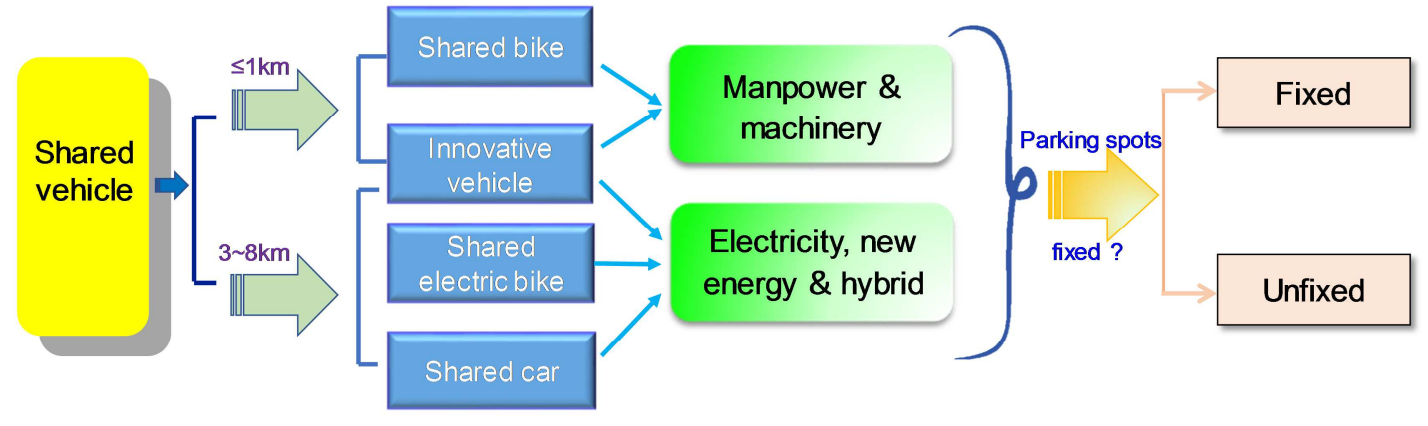

图1 从行程、能源和有无固定停车位角度对国内共享交通工具的分类。

首先可按照续航距离来进行分类, 可大致将其分为可 解决出行者1公里和3 10公里出行需求的共享交通工具。

其中, 共享单车的诞生用“以租代买”、私人盈利企业 提供短时租赁服务的方式, 让民众使用自行车替代大型运 输或私有车辆, 解决了 1 公里内出行的需求, 其次, 根据 单车开锁方式, 可将其分为智能锁共享单车和机械锁共享 单车, 显然, 在互联网时代, 智能锁是更为明智的选择; 另外, 共享单车有无篮和有篮两种款式, 有篮固然方便放 置物品, 但无篮也降低了维修成本, 同时避免了因篮载婴 孩导致的危险。除共享单车外, 体感平衡车、行李箱共享
车和健身代步车等新颖的共享交通工具也应运而生, 解决 了短距离出行的需求。

对于3 10公里出行距离, 其中包含了共享电单车、共 享汽车和部分新型电动代步车。因动力只来源于人的运动, 共享单车无法覆盖到远距离出行的用户，而以电能、新型 能源、人力电力混合发电的交通工具弥补了这一空缺。目 前大多共享电单车用锂电池作为动力, 共享汽车靠汽油运 行, 但有大多数动力电池企业已将电池创新项目提上日程, 另外新型电动代步车如残疾人代步车也能实现远距离行 驶。要有更为严格的管理模式和智能化的充电系统, 为户 
及时规划路线; 从管理层面上来看, 类似共享单车这样无 需固定停车点的交通工具, 则需考虑车辆分配调度、车辆 摆放问题。不管是哪种类型, 共享交通都从不同角度提供 了个性化、快速共享的服务, 拥有较好的市场预期和潜在 价值, 是未来交通一种必然趋势。

\section{3. 能源动力}

共享电单车发展初衷是为了解决3 10公里距离出行 的需求, 在距离上, 它作为共享单车的补充能够辐射到共 享单车辐射不到的用户。共享单车的动力来源于人的运动, 共享电单车一般以电能、新型能源、人力电力混合发电系 统为动力来源, 因此共享电单车对能源动力上就提出了更 高的要求。倘若无法做到持续远距离的行驶, 共享电单车 和共享汽车就失去了意义。故而在本节重点针对非人力驱 动交通工具的能源动力进行综述。

目前的共享电单车能源有多种选择。一般来讲, 电单 车的能源动力是铅蓄电池和锂电池, 汽车靠汽油或者柴油 来驱动, 但近年来新能源的应用层出不穷。使用新型能源, 可以满足我们对共享电单车安全、高续航能力、便捷等的 要求。

共享电单车与共享单车的一字之差意味着能源动力 的差异。在共享电单车在具有更快捷、行驶距离更长的优 点的同时, 我们对其能源动力的要求也更高。作为共享电 单车的核心部件之一, 电池的成本占据着共享电单车整车 成本的 $1 / 3$ 以上, 因此能源动力是共享电单车性能最重要 的考量标准[20]。另一方面, 电池质量的优劣、剩余电量 的精准计算、电池的循环寿命等都决定着共享电单车的用 户体验及盈利效率 $[21]$ 。

以下系统地从新型能源和电能获取装置两个方面综 述了近年来国内外学者提出的可运用于共享交通工具的 能源动力。

\section{1. 新型能源}

电动车一般使用的能源动力为铅蓄电池, 但是铅的污 染较大、能量密度低且过于笨重, 这对于共享交通工具的 推广不利; 锂离子电池系统也是电动车的重要组成部分, 它的工作性能在较大程度上受温度影响, 需要通过设计合 理的热管理系统结构、开发先进热管理控制策略使工作温 度控制在适宜范围内, 从而提高电池性能[22]。而新型能 源动力如太阳能、氢能等则更加环保、便携, 所以在共享 交通工具中新型能源是不二选择。“共享”的本质是充分重 复利用闲置资源，达到高效、低成本和环保的目的。2018 年, 丁宁等[23]以满足我国北京市一年出行需求为功能单 位, 发现平均到每年的单车寿命中, 共享单车碳排放高出 普通单车约11倍。共享电单车和共享汽车所考察的新型能 源区别于普通的电单车锂电池或化石能源, 提倡尽可能得 利用绿色、节能、可持续的新能源。

燃料电池以清洁混合材料为燃料, 绿色环保。 2010 年, J Jia等 [24]研究了以质子交换膜燃料电池 PEMFC (Proton Exchange Membrane Fuel Cell)为主要动力源和超 级电容器 (UC) 作为储能单元的清洁混合动力供电系统
的特性。与具有功率调节单元的传统燃料电池混合系统不 同, 该研究调查了没有 DC/DC转换器的PEMFC和UC混合 系统的电气特性。在评估所提出的系统的平台上, 测试了 基于燃料电池的电动自行车。测试结果表明, 所提出的架 构是有效的, 并取得了良好的效果。通过道路测试, 验证 了 PEMFC可以为负载提供额定功率, 并且UC可以在系统 启动时提供功率, 并在需要时提供峰值功率。

太阳能是自然界最直接的能量形式, 是取之不尽、用 之不竭的理想清洁环保能源, 但是如何高效的转化为机器 可以直接使用的电能才是关键。2017年, Alyona Ivanova 等 [25]提出了一种最佳策略, 用于在连接到不列颠哥伦比 亚省维多利亚州的净计量光伏停车场时协调大型电动车 辆的充电。通过实验说明, 使用CPLEX优化工具(一种可 以将复杂的业务问题表现为数学规划 (Mathematic Programming) 模型的优化工具)将问题归结为混合整数线 性编程问题, 以确定基于季节太阳能潜力的车辆的最佳充 电调度。该算法用于评估最小的馈线容量和安装的充电站 数量, 以及最小化日常运营成本 $(O C)$ 。每个季节都会 进行协调和不协调充电的日常用户单位, 包括和不使用净 计量屋顶光伏装置。确定实施协调充电可以具有高达 $20 \%$ 的OC降低, 而具有协调充电的太阳能发电耦合导致 14 96\% 的降低, 这取决于季节和峰值功率。他们还提出, 在冬季 月份, 通过增加太阳能, $\mathrm{OC}$ 可以随能源成本和能耗的降 低而降低。这是从充分利用新型能源的角度以实现“共享” 得本质。

\section{2. 电能获取装置}

电能产生的来源多种多样, 如何从原材料中获取电能 也是至关重要的技术。传统模式是将电能转化为化学能储 存在电池中, 近年来, 有众多学者提出了新的电能获取装 置, 如开关磁阻 电动机、BLPM (Brushless Permanent-Magnet) 电动机与电磁感应效应和主动诱电技 术等。

2010年, B Bilgin等[26]提出了转子极数大于定子极数 的一种新型开关磁阻电动机 SRM (Switched Reluctance Motor) 配置, 是混合动力辆HEV (Hybrid Electric Vehicle) 和插电式混合动力电动车辆PHEV (Plug-in Hybrid Electric Vehicles)的推进系统的潜在候选者, 它们在恶劣的操作条 件下坚固耐用, 并具有宽的恒定功率范围。在此基础上, 2014年, J Lin等[27]认为高扭矩和功率密度以及开关的低 成本使SRM成为电动自行车的有力候选者, 且证明转子极 弧对转矩性能的影响有限, 它只会导致扭矩脉冲的宽度变 化。但是幅度实际上是相同的 $(0 \mathrm{a})$ 。此外, 介绍了一种 带有 6 个定子极和 10 个转子极的三相外转子SRM, 专为电 动自行车应用而设计, 如 $0 \mathrm{~b}$ 所示, 展示了对齐和未对齐的 转换路径位置的两种情况, 他们通过评估自行车运动学来 启动SRM设计和仿真。

电能获取装置只是有良好的性能而过于笨重的话会 给市民出行带来负担, 这不利于共享交通的推广。2014 年, $\mathrm{YC} \mathrm{Wu}$ 等[28]提出了一种新颖的机电设备, 巧妙地将 外转子无刷永磁BLPM (Brushless Permanent-Magnet)电机 集成到变速器轮毂中, 从而成为紧凑的结构组件, 这使得 
电能获取装置小巧便携。这是一种新颖的设计, 集成了一 个三相、20极/18槽外转子BLPM电机和一个由六连杆、两 个自由度组成的多速传动轮觳, 以克服现有设计的固有缺 陷。并开发了一种基于基本电路的分析方法, 以合成行星 齿轮系的所有齿轮的离合顺序和齿数。集成设备提供六种 前进速度, 包括两个低速驱动、两个直接驱动、两个过载、 以及两种驱动模式, 电动自行车的电动机模式和人力驱动 模式所提出的设计的主要特征是与定子的极靴合并的正 齿轮齿以显着地最小化制动转矩, 该制动转矩是总是引起 BLPM电动机的振动和声学噪声的振荡转矩。其特点是定 子极靴上的齿轮齿不仅具有传动功能, 还可作为虚拟槽, 用于调节BLPM电机的静磁场, 有效降低制动转矩。集成
设计的制动转矩峰值仅为原始BLPM电机的 $9 \%$, 具有相同 的磁铁特性和电机尺寸。这种特征有助于抑制电动自行车 BLPM电机的振动和噪音。

一般电单车的充电需要特定的充电装置, 这对相关部 门的运营维护是一大难点。2017年, 谢炎民[29]提到了无 线充电机应设计 AFC (Automatic Fare Collection System) 自动频率调整电路, 以根据电单车充电过程中的环境温度、 湿度、发射天线和接收天线间隙变化、天线错位、电单车 电池差异、天线参数的等个体差异, 对其电参数做出相应 调整, 以适应大批量的盲配充电, 这使得共享电单车和共 享汽车的充电不需一对一匹配, 让市民使用起来更加便捷, 机构运营维护也更加方便。
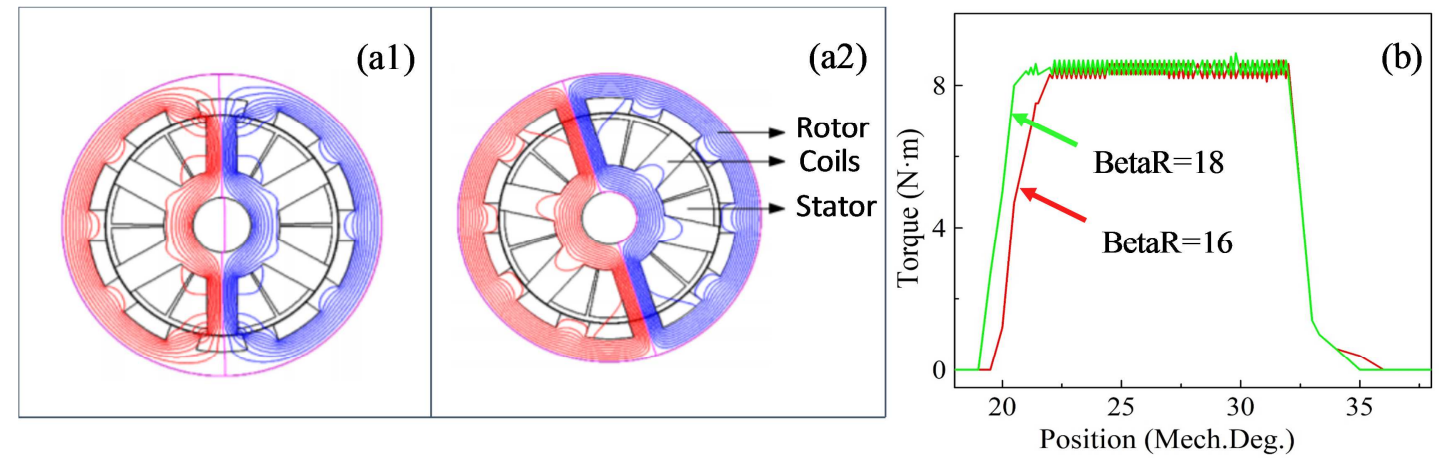

图2 (a1)非对齐的转换路径位置, (a2)对齐的转换路径位置, (b)不同转子极弧的转矩性能比较[26]。

\section{4. 组织与管理}

已大量投入使用的共享单车在一定程度上解决了“最 后1公里”的问题, 但也带来了一些副作用。若没有合理的 管理系统, 将会产生市民出行低效、单车数量分配不合理、 摆放杂乱无章和被恶意损坏偷窃等问题。共享电单车和共 享汽车是伴随着互联网、大数据和人工智能浪潮而产生的, 是与市民出行息息相关的一大创新, 其治理须兴利除弊, 实现绿色低碳、方便出行, 因此要求它们有更完善的高科 技经营模式。

针对可能存在的问题, 我们将从使用和维护两个方面 来进行阐述。

\section{1. 共享交通工具的使用}

共享交通的好处在于它能够实现高效便捷的出行、提 高资源配置率。为践行这一原则, 共享电单车和共享汽车 需要在其使用时做到高效。

\subsection{1. 共享交通工具的充电问题}

除了共享单车目前遇到的问题之外, 共享电单车和共 享汽车需要解决的另一问题就是及时充电。能否合理高效 地充电也是共享电单车能否大量投入的关键。另外, 定点 停车充电也能避免车辆的随意摆放。

那么, 作为充电核心技术的充电桩有什么创新设计呢? 初期, 关于充电桩的创新侧重于健全充电点线下服务系统。 2011年, 孟祥军等 $[30]$ 研制的电动汽车智能充电桩强化了 充电桩电气安全、数据安全设计和环境及电磁兼容性, 电
动汽车充电桩硬件系统主要由主控板、监控板、IC (Integrated Circuit Card) 卡读写器、数字电表、移动通信模 块、触摸屏、指示灯、按键等组成;

软件设计时采用了模块化的编写原则, 既能保证电动 汽车充电桩软件系统高效可靠的运行, 又能使该软件系统 具有良好的扩展性, 对产品升级换代具有非常重要的意义; 带安全存取模块SAM (Secure Access Module)的读卡器采 用硬件加密技术，防止了信息窃取; 充电桩状体采用用交 叉覆盖工艺, 保证恶劣天气适应性, 并具有很好的散热性 和电磁兼容性 $(0)$ 。

近年来, 通过无线WIFI技术、低功耗广域网和物联网 标识模块等现进物联网技术的集成, 充电桩逐渐向智能人 机交互方向过度, 具有较好的社会和经济效益。

2018年，张荣飞等[31]报道了深圳市建立“智慧停车+ 充电一体化”路边充电桩试点项目, 提出了基于互联网的 共享服务电动汽车充电系统。该系统对车载监控系统、微 观网络链接、充电桩监控系统和宏观网络链接的融合, 分 别实现对汽车内部网络对电池状态安全性、剩余电量的监 控、智能推荐系统和互联网平台对充电机系统、配电安全 系统、计费以及网络链接等功能的控制。将底层各系统输 出动态信息并与共享云端连接, 实现了电网、用户与共享 云端的动态调节。另外还分析了该基于互联网的共享服务 充电的理论系统, 提出对充电信息进行集约化、标准化处 理, 充电用户通过 APP可实现预约、排队、请求、即时 结算等。利用大数据进行篮选和处理, 促进了充电站的均 衡使用, 即可以通过共享通信模块实现对充电设施充电信 息的系统化, 实现充电信息的共享, 保证了充电系统的高 效有序运作。 


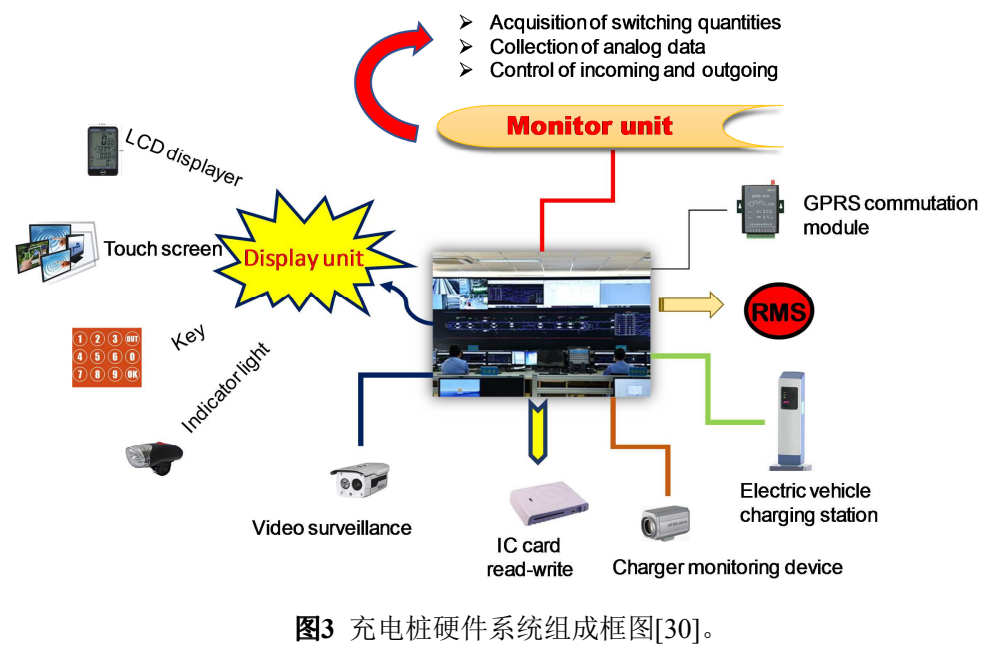

在优化智能充电桩充电系统的基础上, 2018年, X Huang等[32]还考虑到了电动汽车和充电桩之间管理的安 全性。提出了一种基于区域链生态系统中的闪电网络和智 能契约的分散式安全模型，用于IoE (Internet of Energy ) 中的 $\mathrm{EV}$ (Electric Vehicles) 充电管理。提出的模型叫做 LNSC (the Lightning Network and Smart Contract)。整体模 型涉及注册、调度、认证和计费阶段。新提出的安全模型 可以很容易地与当前的调度机制集成, 以增强电动汽车和 充电桩之间的交易安全性。根据现实基础设施的实验结果, 我们的方案可以有效地提高车辆安全性, 该方法可以实现 97 78\%的成功率，综合的基于成本的调度能够实现消费 成本和时间成本间的良好平衡, 这可以为运营商选择合适 的调度策略提供建议。

\subsection{2. 优化配置与调度}

值得一提的是, 虽然共享交通工具的数量已基本饱和, 但资源配置还存在着不合理性。针对资源配置与调度不合 理导致低效出行和交通堵塞的问题, 讨论了配置优化的创 新系统。

\section{1) 车辆配置优化}

2014年，张建国等[33]通过分析一天内不同时段公共 自行车的借还需求情况, 从成本最小化和租赁点满意度最 大化两个目标出发, 在平峰时段建立不带时间窗的公共自 行车车辆调配路径优化模型; 在高峰时段建立基于滚动时
域的公共自行车车辆调配路径优化模型, 并运用蚁群算法 近似求解不同时段车辆调配路径, 解决了公共白行车系统 车辆调度问题。

车辆配置的基础信息在于精准地了解某地区在特定 时间段的人流量及车辆需求量。以下是两个通过聚类技术 方法研究的例子:

2009年, J Froehlich等[34]用数字追踪共享骑自行车系 统, 感知大量数据并加以分析, 揭示人类行为的模式。应 用聚类技术来识别共享跨车站的行为、位置。分析了影响 因素并证实了这一算法的具有一定的预测能力。他们正在 记录 18 个额外的共享自行车计划, 包括巴黎和华盛顿特区, 并准备大规模分析和比较这些城市中共享用户的行为模 式。

2015年，A Sarkar[35]以提高在拥挤城市地区交通方 式高效性和促进公共交通和当地最后一英里连接为目的, 研究了伦敦自行车共享数据集以探索社区结构。使用新颖 的聚类技术得出了独特的行为模式, 揭示了城市同质化的 程度, 并发现异质性只存在于大型系统中。评估了社区互 动和时空动态, 发现地理上孤立和专业化的社区相对一致, 而其余系统表现出波动性, 特别是在高峰时段和周围通勤 时间。通过增加我们对自行共享用户集体行为的理解, 该 分析支持政策评估, 运营决策并促进基础设施设计和管理 的改进 (0)。
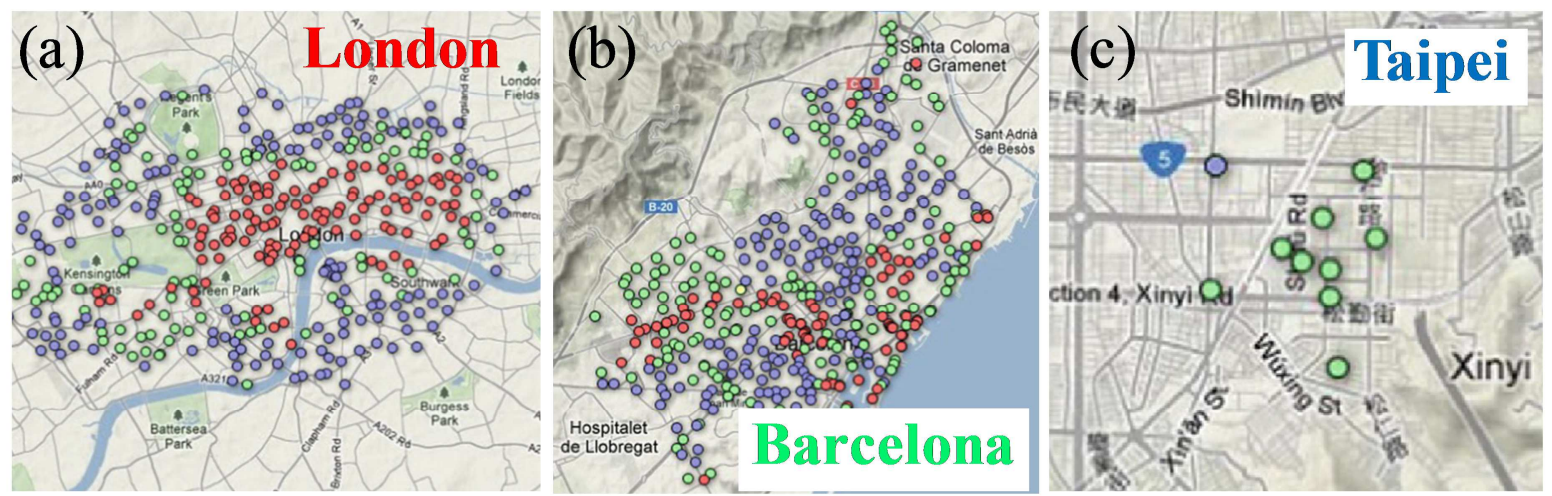

图4 揭示了部分城市同质化程度的聚类图[40]。 


\section{2) 人员配置}

2014年, Junghoon Lee等[36]提出了一种设计和评估 电动汽车共享系统搬迁人员配置性能的方案, 旨在克服库 存不平衡问题, 从而提高服务率。重新定位过程根据未来 的需求估计, 将车辆从溢出站移动到下溢站。对于给定的 目标分布和重新定位, 决定每个群集的工作人员数量以减 少重定位距离和时间。所提出的方案初步运行单位调度程 序, 以最小的人员分配来建立经验距离估计模型。它重复 估计每个集群的重定位成本, 并将工作人员逐个分配给具 有最差重定位距离的集群。性能测量结果表明, 与均匀分 配方案相比, 该方案可以将重定位距离减少多达 $31.7 \%$ 。

\section{2. 维修与回收}

虽共享电单车和共享汽车可弥补短途出行空缺, 发展 前景颇好，但部分地区的政策仍对其采取不鼓励、不支持 的态度。究其原因有, 目前不规范的管理系统、无法保证 交通安全和质量、维修不及时、使用户体验不佳等, 那么 现阶段如何处理好共享电单车和共享汽车的维修和回收 问题呢? 实时跟踪共享交通工具的数据信息对于高效维 修极为重要, 建立一个完善的管家系统亦很关键。

\section{1) 数据信息跟踪}

2014年，金昊炫[37]提到可以通过车联网搭建智能交 通网络, 以此技术作为支撑, 可以使电动汽车服务公司通 过信息端和ABS (Antilock Brake System)系统获取相关车 辆状况信息。借助于信息收集端来获得共享电动汽车的速 度信息, 包含状态的诊断、车辆状态、加速和减速等相关 信息。同时也能够通过这些信息使会员更好的驾驶汽车, 减少会员在驾驶的过程中由于不合理的驾驶习惯而对电 动汽车造成的不良影响。
2017年，YX Zhao等[38]通过对中国HZBike、Mobike 和 ofo 等共享单车最近变化之间的比较, 受到了 IoV (Internet of Vehicle) 的启发, 提出了 IoSB (Internet of Shared Bicycle)的概念和五层模型架构。对中国的IoSB进行了说 明, 涉及信息收集过程、预处理、传输、分析和挖掘。在 数据挖掘部分, 对共享自行车循环数据的几个案例进行数 据分析, 分析了如何开采自行车使用深度学习方法的数据 以及它的困难, 还列出了一些使用IoSB实施有关数据分析 的研究方向。基于位置的服务, 电子围栏和自行车道记录 可以更好地实现。建议将传感器和电池技术嵌入共享自行 车, 产生更多关于骑行的数据。

\section{2) 智能管家系统}

2017 年, 陈雪婷 [39] 提到广州魔动公司推出的 “Morning”系统，意在打造新能源交通工具的全新模式， 用受管理的锂电池共享服务系统, 让锂电池从出厂到回收 全过程使用是共享租借式使用模式, 用户并不需要购买电 池, 电池会在充换电柜里面完成充换电。而且“Morning” 电池智能交换管理共享系统可以实现电池从投入定位和 无线通信模块实现车辆的准确定位和追踪; 通过有效的信 用管理和流程管理, 市场到厂家回收, 全程去向都有系统 数据库跟踪记录, 做到真正意义上的环保。

2017年, 於铉等[40]为了更好地解决共享单车安全防 范措施不完善、维修与回收不便捷、随意停放影响城市管 理秩序等问题，提出了共享单车智能管家系统。采用技术、 信用和管理相结合的方法, 构建了一个硬件终端作为实现 共享单车智能化的基础, 并通过应用传感器技术来解决单 车缺少夜间行车灯、车筐过载检测等安全隐患; 通过GPS 结合骑行者的定位信息、禁停区和电子围栏的设置、用户 信用的管理等, 引导单车有序地停放在电子围栏内 $(0)$ 。

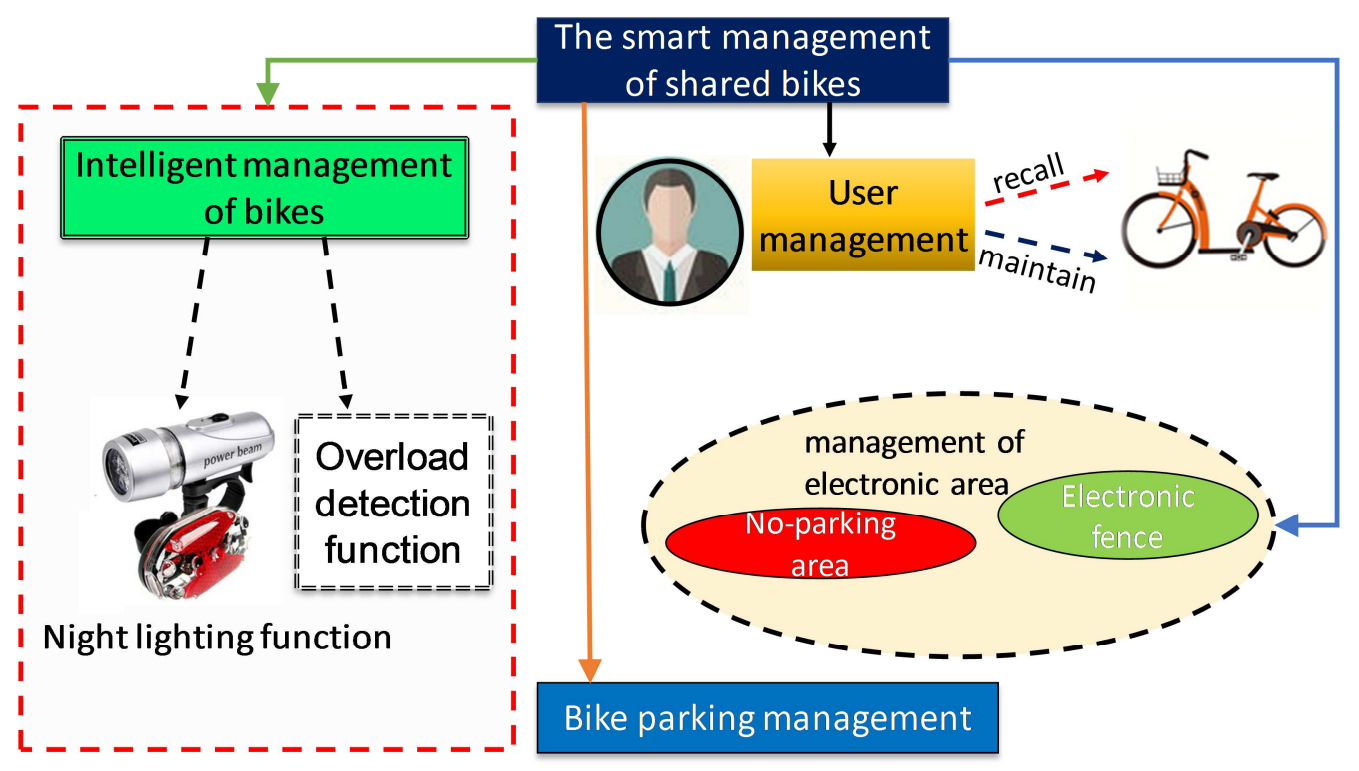

图5 共享单车智能管家系统功能结构框图[40]。 


\section{5. 存在的问题及发展前景}

共享交通工具并不是一个全新的概念，但共享单车的 技术补充、共享电单车和共享汽车的投入发展将会使这一 共享理念得到落实和完善, 尤其是后两者对于方便公众出 行、继续发掘新能源具有独特的优势, 并将对我国的交通、 石油、汽车制造等领域产生深远的影响。对比公众交通出 行方式, 共享出行根据个人的需求与实际情况决定的出行 方式同样具有个性化: 更加自由的出行方式与适合自身需 求的交通工具。目前个性化设置方面需进一步针对不同交 通工具的特点通过创新技术进行完善, 如何让用户真正地 感受到共享出行的便捷还需从使用的各个环节入手, 做到 合理创新、严格把关。要在共享的“共性”前提下，做到“个 性” 的设计与体验还有很多问题亟待解决。另外体感平衡 车、行李箱共享车和健身代步车等新颖的共享交通工具也 可以作为未来共享交通的发展方向。

\section{1) 能量回收}

共享的本质是实现闲置资源的重复有效利用, 减少资 源浪费, 在共享交通领域能量的回收和再利用便是这一思 想的重要体现。传统的铅蓄电池电动车由于能量利用率低 导致电量持续时间短[41]。2016年, 马俊等[42]把电动车 制动产生的摩擦力等机械能转化成电能供蓄电池继续利 用,并采用超级电容作为储能元件, 用这种方法不仅能提高 电动汽车的续航能力而且不容易损害蓄电池的工作寿命。 $\mathrm{YL} \mathrm{Ke}$ 等 $[43]$ 提出的电单车的双象限直流电动机驱动器通 过电枢电流提供再生制动, 从而降低电池存储器的能量消 耗。可见如果能够实现能量回收后重利用, 不仅可以提高 电单车的续航能力, 更能增加传统铅蓄电池的使用寿命。

\section{2) 采取先进防盗措施}

在共享交通中, 用户私藏和破坏车辆的行为使行业蒙 受巨大损失。针对该问题, 可以通过智能锁系统实时查询 指定车辆的具体位置以方便修理和维护。同时, 此举更能 为大数据时代提供有实际意义的市民出行数据用于城市 交通规划。正如摩拜单车宣布已全面支持“北斗 $+\mathrm{GPS}+$ 格 洛纳斯”的三模卫星定位, 定位速度与精度已经达到行业 最高的亚米级 [44], J Chen等[45]设计了一种基于蓝牙低功 耗和GPRS通信的智能锁系统, 笔者认为这都将成为今后 研究重点之一。

\section{3）管理系统规范化问题}

目前的共享交通工具管理系统的规范化程度仍有待 提高, 共享交通工具推广以来, 大部分城市都出现过车辆 摆放杂乱, 车辆分配不合理的情况。去年由于某共享单车 企业的资金监管贵乏而导致押金难退，侵害了众多用户的 权益，导致社会纠纷集中迸发。

车辆的规范化管理未来可参考国内第一例位于成都 的地下共享单车智能停车库和日本东京的地下停车场的 管理模式, 人性化和智能化的管理方便了大众的出行, 同 时解决了无序摆放的问题，另外，基于未来居民区的规范 化, 共享交通工具的管理和小区管理的结合不失为一个好 的方案, 当用户数量波动不大时, 居民在保持骑行频率的 前提下可考虑申请固定停放点, 为车辆宏观分配数量提供 依据; 车辆的分配与调度目前仍停留在较为机械的方式, 共享交通工具调度过程中的运输方式、运输地点选择和运
输频率判断可考虑与物联网共享系统结合, 这是值得研究 与挖掘的技术创新点。

又如前述, 尤其对于共享单车与共享电单车, 智能锁 是不可获取的部分。设计卓越的智能锁不仅可以解决车辆 的防盗问题，还为大数据体系提供市民出行数据。2018 年, 卢泽华[46]提出共享单车已成为少数形成规模化连接 数的物联网终端, 而这个“连接”的核心就是智能锁。智能 锁背后连接着庞大的数据库和人工智能平台, 从而形成一 条完整的“数据链”，收集使用前中后三个阶段的数据，方 便日后对单车投放数量和区域进行精准分配, 同时解决了 乱停单车带来的城市公共交通管理问题。智能锁通过卫星 系统、蓝牙技术等可以记录收集市民的出行规律，为城市 的交通规划提供真实可靠的数据, 这也是规范化管理的发 展方向之一。

\section{4）政策导向及法律的完善}

基于“重资产”的运营模式，若没有政府政策或资金支 持，不仅难以吸引运营方和投资方，后期用户押金返还、 车辆处置等也会成为社会问题, 导致企业经营风险高、退 出机制不完善。目前法规政策建设滞后于技术创新, 且缺 乏一套完善的用车规范或法律条例以避免破坏车辆或影 响其他消费者的使用。市场发展存在一定盲目性, 且发展 不平衡: 我国共享单车已在大城市基本饱和, 但在中小型 城市尚不能满足居民出行需求, 而共享电单车处于发展瓶 颈阶段, 共享汽车则尚在起步阶段。这些问题亟需通过政 府政策引导, 从而促进共享交通行业健康有序发展。

\section{6. 结束语}

总之, 近年来共享交通技术的升级, 管理体系的完善 及“互联网+”的快速发展使得共享交通发展迅猛，共享交 通是互联网、大数据技术的发展载体, 也是共享经济在交 通领域的表现形式, 具有无限的发展潜力, 共享交通将是 未来交通的大势所趋。但受到续航能力、地理环境、管理 系统覆盖面的影响而存在盲区。如长途交通出行时, 共享 电单车和共享汽车的充电问题有待解决; 共享交通工具在 结构和技术上的创新, 如减振结构、防雨电池等对共享交 通工具的进一步推广也具有重要的意义; 针对各种复杂的 交通运输方式及技术，如何对它们进行适当分类、确立性 能指标, 并制订出各类测试标准及其工程应用规范等, 将 是今后研究的重要方向之一。目前, 共享单车处于瓶颈期, 共享电单车尚未全面推广, 共享汽车正在起步, 管理系统 也存在本文前述的部分问题, 该行业还有很大的发展空间 与可挖掘的技术创新点。共享交通的重要意义在于较高的 参与度和易获得性, 这也是共享交通工具的共同特性。运 营商和第三方数据平台合作, 能充分利用数据库为用户规 划路径, 并能实现数据共享。新的政策和监管已逐渐为各 种机遇敞开大门, 给共享交通工具的发展带来了无限的发 展潜力。当然, 这些共同特性所带来的还有痛点和难题。 不论是单车、汽车, 还是各类独具个性的交通工具, 都会 涉及到停放在哪里、押金怎么管、出现事故怎么划分责任 等共性问题。期望本文能对共享交通后续的发展起到抛砖 引玉之用; 也期望在不久的将来, 完善的共享交通体系能 
够改进我们的生活方式，实现便捷、个性化、绿色、安全 的出行。

\section{参考文献}

[1] 马强. 共享经济在我国的发展现状、瓶颈及对策[J]. 现代经 济探讨, 2016(10):20-24。

[2] 田华. 探究当代共享经济发展中的利与弊[J]. 中国战略新 兴产业, 2017(48)。

[3] 周章荣,蔡慧贤,何妍,曹金龙.共享单车的现状分析及管理建 议[J].中国集体经济,2018(33):86-88。

[4] YL Ke, YC Chuang, HS Chuang, Energy Recovery Electric Bicycle with Two-Quadrant DC Motor Drivers[J]. Industry Applications Society Meeting, 2009: 1-7.

[5] Zervas G, Proserpio D, Byers J. The Rise of the Sharing Economy: Estimating the Impact of Airbnb on the Hotel Industry [J]. Social Science Electronic Publishing, 2013.

[6] L Caggiani, M Ottomanelli, A Dynamic Simulation based Model for Optimal Fleet Repositioning in Bike-sharing Systems [J]. Procedia - Social and Behavioral Sciences, 2013. 87: 203-210.

[7] 汤天波，吴晓隽. 共享经济: “互联网+”下的颠覆性经济模 式[J]. 科学发展, 2015(12):78-84。

[8] 卢希鹏.随经济:共享经济之后的全新战略思维 [J].人民论 坛·学术前沿,2015(22):35-44。

[9] 孟凡新. 共享经济模式下的网络交易市场治理:淘宝平台例 证[J]. 改革, 2015(12):104-111。

[10] 李强治. 共享经济的本质:物尽其用的经济学分析 [J]. 电信 网技术, 2016(12):32-35。

[11] Kai-way Chun, Zih-Hong Chen, Chi-Chia Sun, IOT solution for public bicycle system [J]. IEEE International Conference on Consumer Electronics-TaiWan , 2016: 1-2.

[12] 陈元志. 面向共享经济的创新友好型监管研究 $[J]$. 管理世 界, 2016(8):176-177。

[13] Mason S G. Retail Gravitation and Economic Impact: A Market-Driven Analytical Framework for Bike Share Station Location Analysis in the U.S. [J]. International Journal of Sustainable Transportation, 2016, 10 (3): 247-259.

[14] 刘蕾, 鄢章华. 共享经济—从"去中介化"到"再中介化" 的被动创新 [J]. 科技进步与对策, 2017, 34(7):14-20。

[15] J Chen, X Jiang, Design of Sharing Bicycle Smart Lock System Based on Bluetooth and GPRS Communications[J]. International Conference on Vehicle, Mechanical and Electrical Engineering, 2017 (04).

[16] Tao Y, Ziyi L I, Yang L, et al. Research on Bicycle Sharing Economy-Based on Fuzzy Comprehensive Evaluation Method [J]. Journal of Jiujiang University, 2018.
[17] 毛方强、杨华、方勇、郑寅, 夏添. 基于公共自行车的共 享电踏车行业分析与系统实现 $[\mathrm{J}]$. 物联网技术, 2018(7)。

[18] 黄砷才、蓝伟杰、李国武、邓礼铭, 严春时. 基于RFID技 术的电动车防盗系统 [J]. 科技视界, 2018(16)。

[19] 彭华涛, 罗一郎, 夏德. 共享经济创业的异常模仿行为及 其协同治理[J]. 科学学研究, 2018(7)。

[20] 汤桃峰. 电动车锂离子电池组 SOC预估及一致性研究 [D]. 重庆大学, 2011。

[21] 柯南极, 张玉良, 饶沝涛, et al. 电动汽车无线充电位置的 对准方法和系统。

[22] 丹聑, 姚程宁, 张扬军, 等. 基于热管技术的动力电池热管 理系统研究现状及展望. 科学通报, 2019, 64: 682-693。

[23] 丁宁、杨建新、逯馨华, 刘晶茹. 共享单车生命周期评价 及对城市交通碳排放的影响——以北京市为例 $[\mathrm{J}]$. 环境科 学学报。

[24] J Jia, G Wang, YT Cham, Y Wang, M Han, Electrical Characteristic Study of a Hybrid PEMFC and Ultracapacitor System [J]. IEEE Transactions on Industrial Electronics, 2010. 57 (6): 1945-1953.

[25] Alyona Ivanova, Julian ALberto Fernandez, Curran Crawford, Ned Djilali, Coordinated char M Corno, D Berretta, P Spagnol, SM Savaresi, Design, Control, and Validation of a Charge-Sustaining Parallel Hybrid Bicycle [J]. IEEE Transactions on Control Systems Technology, 2016. 24 (3): 817-8297.

[26] B Bilgin, A Emadi, M Krishnamurthy, Design considerations for switched reluctance machines with higher number of rotor poles for solar-assisted plug-in electric auto rickshaw [J]. IEEE International Symposium on Industrial Electronics, 2010: $1247-1252$.

[27] J Lin, N Schofield, A Emadi, External-rotor 6-10 switched reluctance motor for an electric bicycle $[\mathrm{J}]$. Conference of the IEEE Industrial Electronics Scieology, 2014. 1 (4): 348-356.

[28] YC Wu, WH Hsieh, BW Lin, Design of a six-speed transmission hub with an integrated brushless permanent-magnet motor used for electric bicycles [J]. Engineering Computations, 2014. 31 (2): 160-176.

[29] 谢炎民. 浅谈共享电单车无线充电技术 $[J]$. 中国自行车, 2017(5): 64-65。

[30] 孟祥军、梁涛、王兴光、陈杰, 李建祥. 电动汽车智能充 电桩的设计与实现 $[J]$. 信息技术与信息化, 2011(6): 58-61。

[31] 张荣飞、官子超、陈锐、张荣华，覃飞. 基于共享服务的 电动汽车充电系统设计与实现 [J]. 科学技术创新, 2018(10)。

[32] X Huang, C Xu, P Wang, H Liu, LNSC: A Security Model for Electric Vehicle and Charging Pile Management based on Blockchain Ecosystem [J]. IEEE Access, 2018. PP (99): 1-1.

[33] 张建国、吴婷, 蒋阳升. 基于蚁群算法的公共自行车系统 调度算法研究 [J]. 西华大学学报:自然科学版. 2014, 33(3): 70-76。 
[34] J Froehlich, J Neumann, N Oliver. Sensing and Predicting the Pulse of the City through Shared Bicycling [J]. International Joint Conference on Ijcai. 2009, 38 (4): 1420-1426.

[35] A Sarkar, N Lathia, C Mascolo, Comparing cities' cycling patterns using online shared bicycle maps [J]. Transportation, 2015. 42 (4): 541-559.

[36] Junghoon Lee, Gyung-Leen Park, Design of a Relocation Staff Assignment Scheme for Clustered Electric Vehicle Sharing Systems [J]. Computational Science and Its Applications, 2014.

[37] 金昊炫. 车联网对电动汽车共享服务的支持 [J].电子技术与 软件工程, 2017(21): 248-248。

[38] YX Zhao, YS Su, YC ChangA. Real-Time Bicycle Record System of Ground Conditions based on Internet of Things[J]. IEEE Access, 2017, PP (99): 1-1.

[39] 陈雪婷. 打破行业桎梏推动新能源发展一一全国首个电动 车共享电池"Morning"系统问世 $[\mathrm{J}]$. 电动自行车, 2017(9): 19-21。
[40] 於铉、张洁, 张明. 共享单车智能管家系统的设计与实现 $[\mathrm{J}]$. 软件, 2017, 38(10): 194-197。

[41] 刘文军. 混合动力车用软开关双向DC-DC变换器设计[D]. 西南交通大学, 2016。

[42] 马俊, 于士军. 电动汽车制动能量回收实验设计 $[\mathrm{J}]$. 河北 农机, 2016(9):64-65。

[43] YL Ke, YC Chuang, HS Chuang, Energy Recovery Electric Bicycle with Two-Quadrant DC Motor Drivers[J]. Industry Applications Society Meeting, 2009: 1-7.

[44] 耿炎、徐燕华, 包莹莹. 基于物联网技术的共享单车发展 趋向分析 [J]. 无线互联科技, 2017(22): 12-13。

[45] J Chen, X Jiang, Design of Sharing Bicycle Smart Lock System Based on Bluetooth and GPRS Communications [J]. International Conference on Vehicle, Mechanical and Electrical Engineering, 2017 (04).

[46] 卢泽华. 小智能锁牵大数据链 $[\mathrm{J}]$. 电动自行车, 2018(1): 19-20。 